\title{
Maffucci Syndrome with Hemangioma of the Liver
}

\author{
Raffaele Pezzilli Carla Serra Paola Tomassetti \\ Emilio Brocchi Davide Campana Roberto Corinaldesi
}

Department of Internal Medicine and Gastroenterology, Sant'Orsola-M. Malpighi

Hospital, University of Bologna, Bologna, Italy

\section{Key Words}

Enchondromatosis · Hemangioma · Magnetic resonance imaging · Contrast-enhanced ultrasonography · Doppler

\begin{abstract}
The presence of visceral hemangiomas in the syndrome of multiple enchondromas and subcutaneous hemangiomas, also named Maffucci syndrome, is exceedingly rare; until now noncutaneous hemangiomas have been described in 4 patients: they were found in the tongue in one patient, in the oral cavity in the second patient, in both the oral cavity and the colon in the third patient, and in the spleen in the fourth patient. We report the first case of hemangioma localized in the liver in a patient with Maffucci syndrome; furthermore, due to the impossibility of carrying out an MRI to define the hepatic lesion, an ultrasonographic real-time perfusion imaging study with a contrast agent was performed.
\end{abstract}

\section{Introduction}

The syndrome of multiple enchondromas and subcutaneous hemangiomas, known as Maffucci syndrome, is very rare, accounting for about 200 cases during the last 140 years [1]. The presence of visceral hemangiomas in this disease is exceedingly rare; for this reason we believe it to be of interest to report a case of hemangioma localized in the liver in a patient with Maffucci syndrome. To our knowledge, this is the first description of the presence of a hemangioma localized in the liver in a syndrome of multiple enchondromas and subcutaneous hemangiomas.

\section{Case Report}

B.M. is a 51-year-old female affected by Maffucci syndrome with skeletal deformities involving both arms, the left scapula and chest and having variable sized deep bluish nodules on the fingers of both hands. The first lesion localized on the left arm was noted at the age of 6 years. At the age of 30 years she 
underwent surgery for the rupture of the vascular lesion localized at the 5th left finger; the histology of the surgical specimen showed multiple blood-filled cavities lined with a single layer of endothelial cells. The patient was also operated at the age of 40 years because of a chondrosarcoma of the left humerus. She is also affected by hypertension, for which she is under pharmacological treatment with beta blockers and diuretics. The patient was admitted to our hospital 15 days after an ultrasound examination performed as a routine follow-up for her disease. This examination showed a hepatic lesion which had never been identified in previous follow-ups. On admission, the patient was asymptomatic and clinical examination did not reveal any alterations apart from those compatible with her disease, namely limitation of movement and chest abnormalities. The patient had no mental or psychiatric abnormalities. Her blood pressure was $130 / 80 \mathrm{~mm} \mathrm{Hg}$ and no alterations were observed in the electrocardiogram. Routine blood analysis revealed an increase of GGT (124 U/1, normal values <50 U/l) and of alkaline phosphatase concentrations (330 U/l, normal values 98-280 U/l). Chest X-ray showed postsurgical alterations of the left shoulder and irregularly shaped, radiolucent areas with stippled calcification within the right humerus; costal dyschondroplasias were also found. Ultrasonographic examination confirmed a dishomogenous neoformation having a size of $11 \times 10 \mathrm{~cm}$ localized at the IV hepatic segment with hyperisoechogenic pattern and several anechogenic areolae (fig. 1); ultrasonographic color Doppler examination of this lesion showed peripheral arterial vascular signals. An ultrasonographic real-time perfusion imaging study was performed with an Esatune equipment (Esaote S.p.A., Genoa, Italy) using a convex probe of $3.5 \mathrm{MHz}$ in order to better define the hepatic lesion. The Esatune was coupled with the CnTI (contrast tuned imaging) technique, which is a combined hardware and software product for imaging enhancement. A second generation of contrast agent (SonoVue, Bracco International B.V., Amsterdam, The Netherlands) was also used: a bolus of $2.5 \mathrm{ml}$ of SonoVue was rapidly injected i.v. in the left antecubital vein using a $20 \mathrm{G}$ needle; this bolus was immediately followed by i.v. injection of a bolus of $10 \mathrm{ml}$ of saline solution. This examination showed, during the arterial phase, a globular enhancement at the periphery of the lesion with progressive centripetal fill-in in the portal and parenchymal phase (fig. 2). Because the patient had an internal orthopedic device, we did not perform a MRI; in order to confirm the ultrasonographic findings, a spiral computed tomography with i.v. contrast medium was performed. At the IV liver segment the computed tomography examination showed an area with a centripetally advancing border of enhancement; this area then became progressively and homogeneously hyperdense. The patient was discharged from our department with the diagnosis of hepatic hemangioma and one month later a new ultrasonographic examination showed that the dimension of the hepatic hemangioma was unchanged.

\section{Discussion}

The true relationship between Maffucci syndrome and chromosomal abnormalities is still not fully known. This syndrome appears to be unrelated to race and sex and patients are usually normal at birth. The presenting symptoms may be skeletal or vascular and usually occur in infancy as in our patient. The extent of skeletal involvement is variable in enchondromatosis and may include dysplasia which is not directly attributable to enchondromas. Enchondromas are usually in close proximity to, or in continuity with, growth plate cartilage. Consequently, they may result from abnormal regulation of proliferation and terminal differentiation of chondrocytes in the adjoining growth plate. The osseous lesions most frequently involve the phalanges, metacarpals and metatarsal. Maffucci syndrome may be associated with three types of vascular lesions: cavernous hemangiomas, phlebectasias and lymphangiectasias-lymphangiomas [2]. Clinical problems caused by enchondromas include skeletal deformity and the potential for malignant change reported in approximately $30 \%$ of reported cases, with chondrosarcomas being the most common: our patient developed this latter complication at the age of 40 years when she was operated on for a chondrosarcoma localized in the left humerus. However, involvement of the digestive system and the abdominal organs has rarely been reported in patients with Maffucci syndrome: until now hemangiomas of the tongue have been described in one patient [3], in the oral cavity in a second patient [4], in both the oral cavity and the colon in a third patient [2], and in the spleen in a fourth patient [5]. The case we report is the first description of a hepatic hemangioma in a patient with Maffucci syndrome. Another characteristic of our case is that the diagnosis of 
hemangioma of the liver was made using a real-time perfusion imaging ultrasound with a contrast agent, whereas MRI was carried out to diagnose the hemangioma of the spleen in the case reported by Ahmed et al. [5]. We believe that real-time perfusion imaging with an ultrasound contrast agent is another possible technique in diagnosing hemangioma localized in abdominal parenchymal organs in patients with Maffucci syndrome, especially in those patients in whom MRI cannot be carried out.

Fig. 1. Standard ultrasonographic image of the hepatic hemangioma. Horizontal and vertical lines indicate hemangioma diameters.

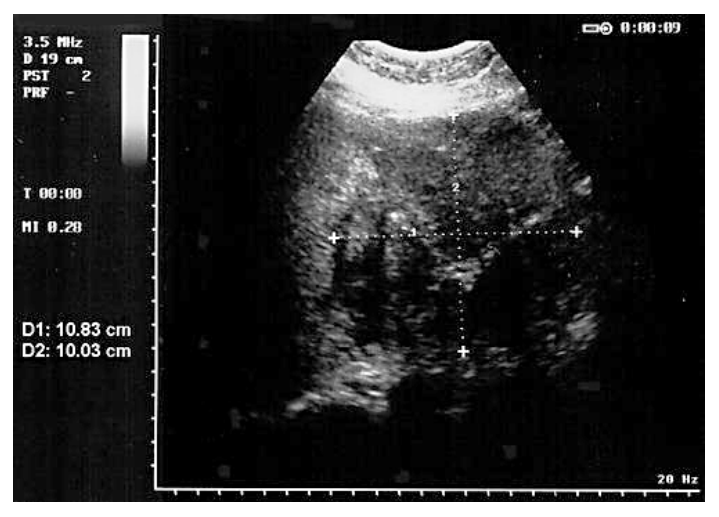

Fig. 2. Ultrasonographic real-time perfusion imaging of the hepatic hemangioma (indicated by the arrowheads) with a contrast agent. a One minute after contrast agent infusion (end of the arterial phase). b Three minutes after contrast agent infusion.
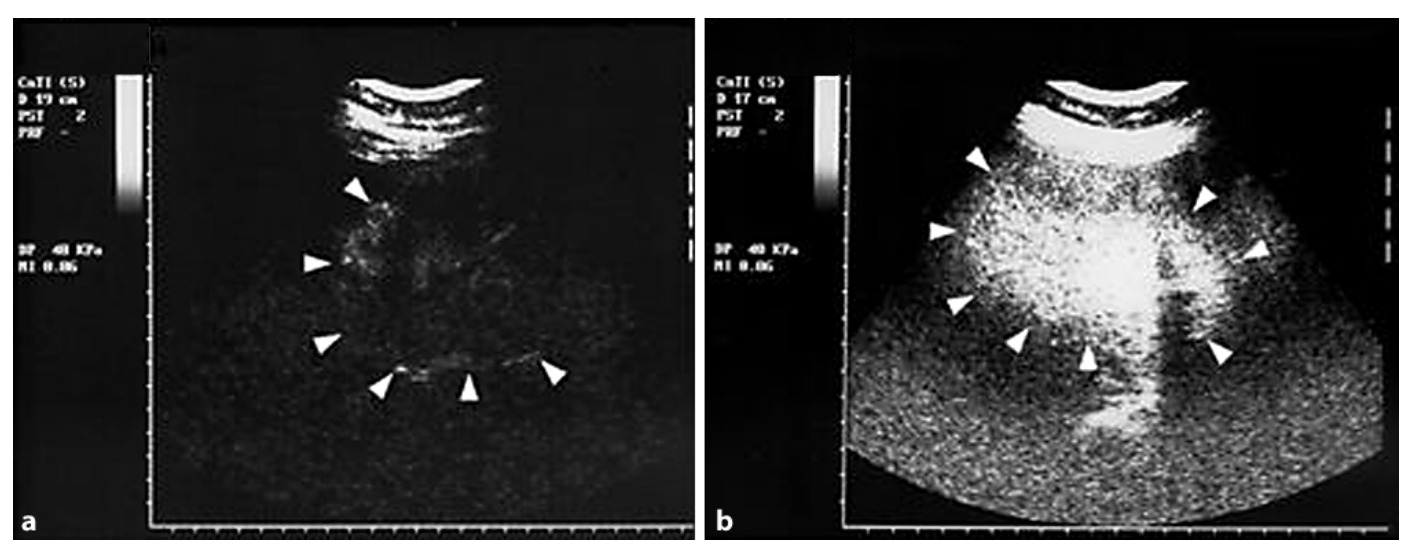


\section{References}

1 Babala J, Horn F, Plank L, Szepe P, Cingel V, Vidiscak M, Studeny S, Siman J: Maffucci's syndrome: case report of a 5-year-old female patient. Rozhl Chir 2002;81:88-92.

-2 Lee NH, Choi EH, Choi WK, Lee SH, Ahn SK: Maffucci's syndrome with oral and intestinal haemangioma. Br J Dermatol 1999;140:968-969.

-3 Laskaris G, Skouteris C: Maffucci's syndrome. Report of a case with oral hemangiomas. Oral Surg Oral Med Pathol 1984;57:263-266.

4 Wolf M, Engelberg S: Recurrent oral bleeding in Maffucci's syndrome: report of a case. J Oral Maxillofac Surg 1993;51:596-597.

5 Ahmed SK, Lee WC, Irving RM, Walsh AR: Is Ollier's disease an understaging of Maffucci's syndrome? J Laryngol Otol 1999;113:861-864. 